\title{
Movimentação de nitrato em horizonte superficial e subsuperficial de Latossolo e Nitossolo com cargas variáveis
}

\author{
Marco Aurélio Kondracki de Alcântara(1) e Otávio Antonio de Camargo ${ }^{(2)}$
}

\begin{abstract}
(1)Universidade de São Paulo, Escola de Engenharia de Lorena, Departamento de Biotecnologia, Caixa Postal 116, CEP 12602-810 Lorena, SP. E-mail: marko@usp.br ${ }^{(2)}$ Instituto Agronômico, Centro de Pesquisa e Desenvolvimento em Solos e Recursos Agroambientais, Avenida Barão de Itapura, no 1.481, Caixa Postal 28, CEP 13020-902 Campinas, SP. E-mail: ocamargo@iac.sp.gov.br
\end{abstract}

\begin{abstract}
Resumo - O objetivo deste trabalho foi avaliar a movimentação de nitrato em colunas de solo considerando-se, principalmente, a quantidade total recuperada, o ajuste entre as curvas de transposição experimental e predita por um modelo matemático e os fatores de retardamento obtidos pelo modelo. Foram utilizadas amostras dos horizontes A e B de um Latossolo Vermelho acriférrico e de um Nitossolo Vermelho eutrófico (LV-A, LV-B, NV-A e NV-B). O experimento de lixiviação foi conduzido tendo em vista a teoria do deslocamento miscível, utilizando como pulso $100 \mathrm{~mL}$ de $\mathrm{KNO}_{3} 10 \mathrm{mmol} \mathrm{L}^{-1}$. As quantidades lixiviadas de nitrato e recuperadas nas colunas, para os solos NV e LV nos horizontes A e B, variaram de 0,405 a 1,432 $\mathrm{mmol} \mathrm{L}^{-1}$. Houve correlação significativa $(\mathrm{p}<0,05)$ entre as curvas experimentais e as ajustadas pelo modelo para as colunas NV-A, NV-B e LV-B. O modelo matemático utilizado é adequado para prever a lixiviação de nitrato em colunas do horizonte B dos solos Nitossolo Vermelho Eutrófico e Latossolo Vermelho Acriférrico. Nas colunas dos solos com bom ajuste entre dados experimentais e preditos, o fator de retardamento é maior do que 1 , o que indica que a movimentação do nitrato é retardada em relação ao avanço da frente de molhamento do solo.
\end{abstract}

Termos para indexação: fator de retardamento, modelo matemático, solos tropicais.

\section{Nitrate movement on surface and subsurface horizons of Udox and Udult soils with variable charges}

\begin{abstract}
The objective of this work was to evaluate nitrate movement on soil columns considering mainly, total amount recovered, fit among experimental and model-predicted transposition curves, and model-obtained retardation factors. Samples of A and B horizons of Udox and Udult soils were used. A leaching experiment was carried out considering the miscible displacement theory, using $100 \mathrm{~mL}$ of a $10-\mathrm{mmol} \mathrm{L}^{-1} \mathrm{KNO}_{3}$ solution as pulse. The amounts of nitrate leached and recovered in the columns for Udox and Udult soils in horizons A and B varied from 0.405 to $1.432 \mathrm{mmol} \mathrm{L}^{-1}$. There was significant $(\mathrm{p}<0.05)$ correlation between experimental and model-predicted curves for columns Udult-A, Udult-B and Udox-B. The mathematical model used is suitable for predicting nitrate lixiviation in B-horizon columns in Udox and Udult soils. The retardation factor was greater than 1.0 in the soil columns with good fit among experimental and predicted data, which indicates that the nitrate movement is delayed in comparison to the advance of the soil wetting front.
\end{abstract}

Index terms: retardation factor, mathematical model, tropical soils.

\section{Introdução}

A movimentação de nitrogênio no solo constitui um potencial de risco de contaminação de águas subterrâneas. Alcântara \& Camargo (2005) verificaram adsorção de nitrato por cargas positivas do solo, o que retardou sua movimentação em relação ao avanço da água. Donn \& Menzies (2005) estudaram a lixiviação de nitrato em subsolos profundos e ricos em óxidos de ferro e observaram retardamento da lixiviação proporcional à quantidade de cargas positivas do solo.

Os teores recuperados de vários elementos no líquido percolado por meio de colunas de solo têm sido utilizados em alguns trabalhos para quantificar a sua movimentação (Oliveira et al., 2001; Prata et al., 2003; Alcântara \& Camargo, 2004; Barizon et al., 2006; Doltra \& Muñoz, 2010). Mais recentemente, tem-se estudado a movimentação utilizando-se modelos físico-matemáticos (Borges Júnior \& Ferreira, 2006; Trivedi \& Babadagli, 2008; Fonseca et al., 2009; Rose et al., 2009). Tais modelos são resultantes de soluções da equação diferencial de transporte de solutos no solo em relação ao avanço da interface entre o líquido deslocador e o deslocado, e têm como base, fluxo de massa, dispersão, difusão e retardamento dos solutos. 
Programas computacionais aplicados a dados experimentais oriundos de ensaios de deslocamento miscível em colunas constituem ferramentas para o estudo da dinâmica de solutos no solo (Melo et al., 2006). Existem diversos aplicativos computacionais, como o STANMOD (Simunek et al., 2008), que, além de verificar o ajuste entre os dados experimentais e os simulados pelo modelo, fornece dois importantes parâmetros de transporte: o fator de retardamento (FR) e o coeficiente de dispersão-difusão (D).

O objetivo deste trabalho foi avaliar a movimentação de nitrato em colunas constituídas pelo horizonte superficial e subsuperficial de um Nitossolo e de um Latossolo e a aplicação de um modelo matemático aos dados obtidos.

\section{Material e Métodos}

Foram utilizadas amostras dos horizontes A e B de um Nitossolo Vermelho eutrófico (NV) e de um Latossolo Vermelho acriférrico (LV) (Santos et al., 2006) - aqui denominadas de NV-A, NV-B, LV-A e LV-B, com a indicação do solo e do horizonte a que se referem -, coletadas no município de Ribeirão Preto, SP $\left(21^{\circ} 05^{\prime} \mathrm{S} 47^{\circ} 36^{\prime} \mathrm{W}\right)$.

As amostras foram coletadas nas profundidades de 0-20 e de 50-70 cm, secadas ao ar, tamisadas a $2 \mathrm{~mm}$, homogeneizadas e armazenadas. A deformação das amostras de solo altera a estrutura original do solo encontrada na natureza. Esse procedimento possibilita minimizar possíveis efeitos de fendas e caminhos preferenciais ao deslocamento de solutos pelo solo, o que alteraria de modo significativo o seu transporte. Além disso, a homogeneização do solo possibilita a incorporação de corretivos e fertilizantes às amostras, de modo semelhante ao que aconteceria em operações convencionais de preparo do solo no campo. Os atributos químicos e físicos das amostras utilizadas, determinados de acordo com o método de Camargo et al. (1986), encontram-se na Tabela 1.

Foram utilizadas colunas de percolação para o estudo da movimentação do nitrato, tendo em vista a teoria do deslocamento miscível, descrita detalhadamente em Reichardt \& Timm (2004). O método utilizado na condução das colunas foi adaptado de Alcântara \& Camargo (2001). As colunas de percolação utilizadas foram feitas de acrílico, com $0,056 \mathrm{~m}$ de diâmetro interno e 0,30 m de altura. Para a manutenção de fluxo constante na coluna, utilizou-se uma bomba peristáltica. A coleta das amostras no efluente foi feita a intervalos regulares, com um coletor de frações automático. Foi utilizada como pulso de nitrato uma solução $10 \mathrm{mmol} \mathrm{L}^{-1}$ de $\mathrm{KNO}_{3}$ e como solução deslocadora, $\mathrm{CaCl}_{2} 50 \mathrm{mmol} \mathrm{L}^{-1}$. A sequência de aplicação de soluções consistiu resumidamente das seguintes etapas: 1 , saturação lenta da coluna a partir da base, com solução deslocadora; 2, aplicação da solução deslocadora a partir do topo da coluna, até obtenção de um fluxo constante; 3, aplicação do pulso de nitrato; e 4, aplicação de solução deslocadora novamente. A quantificação das concentrações (C) de nitrato foi feita nas amostras dos efluentes coletados durante as etapas 3 e 4, utilizando-se o método colorimétrico pela reação do nitrato com brucina em presença de ácido sulfúrico (Baker, 1967). Para cada solo foi conduzida uma coluna, sem emprego de repetição, uma vez que o interesse fundamental do trabalho é avaliar qualitativamente a tendência do fenômeno.

Foram obtidas curvas de transposição do elemento em cada coluna conduzida. O volume de solução aplicado em relação àquele ocupado pelos poros do solo na coluna $\left(\mathrm{V} / \mathrm{V}_{0}\right)$ foi registrado na abscissa e, na ordenada,

Tabela 1. Caracterização química e física dos solos Nitossolo Vermelho eutrófico (NV) e Latossolo Vermelho acriférrico (LV) nos horizontes A e B.

\begin{tabular}{|c|c|c|c|c|}
\hline \multirow[t]{2}{*}{ Atributo } & \multicolumn{2}{|c|}{ NV } & \multicolumn{2}{|c|}{ LV } \\
\hline & A & B & A & $\mathrm{B}$ \\
\hline $\mathrm{pH} \mathrm{CaCl} 2$ & 5,3 & 5,5 & 4,6 & 5,0 \\
\hline P resina $\left(\mathrm{mg} \mathrm{dm}^{-3}\right)$ & 9 & 5 & 15 & 3 \\
\hline $\operatorname{MO}\left(\mathrm{g} \mathrm{kg}^{-1}\right)^{(1)}$ & 46 & 12 & 29 & 10 \\
\hline $\mathrm{Al}\left(\mathrm{mmol}_{\mathrm{c}} \mathrm{dm}^{-3}\right)$ & 0,0 & 0,0 & 4,0 & 0,0 \\
\hline $\mathrm{H}+\mathrm{Al}\left(\mathrm{mmol}_{\mathrm{c}} \mathrm{dm}^{-3}\right)$ & 42,0 & 26,0 & 52,0 & 28,0 \\
\hline $\mathrm{SB}\left(\mathrm{mmolc}_{\mathrm{dm}}^{-3}\right)^{(2)}$ & 95,3 & 30,7 & 14,5 & 5,3 \\
\hline $\mathrm{CTC}_{\mathrm{pH} 7,0}\left(\mathrm{mmol}_{\mathrm{c}} \mathrm{dm}^{-3}\right)$ & 137,3 & 56,7 & 66,5 & 33,3 \\
\hline $\mathrm{CTC}_{\mathrm{pH}}\left(\mathrm{mmol}_{\mathrm{c}} \mathrm{dm}^{-3}\right)^{(3)}$ & 104,3 & 36,4 & 22,5 & 11,0 \\
\hline $\mathrm{CTA}_{\mathrm{pH}}\left(\mathrm{mmol}_{\mathrm{c}} \mathrm{dm}^{-3}\right)^{(3)}$ & 5,1 & 4,8 & 3,1 & 7,8 \\
\hline $\mathrm{CL}\left(\mathrm{mmol}_{\mathrm{c}} \mathrm{dm}^{-3}\right)^{(4)}$ & $-99,2$ & $-31,6$ & $-19,4$ & $-3,2$ \\
\hline $\mathrm{PESN}^{(5)}$ & 3,56 & 3,22 & 3,24 & 4,94 \\
\hline Fe oxalato $\left(\mathrm{g} \mathrm{kg}^{-1}\right)^{(6)}$ & 14,2 & 8,5 & 4,9 & 1,8 \\
\hline Al oxalato $\left(\mathrm{g} \mathrm{kg}^{-1}\right)^{(6)}$ & 2,6 & 3,4 & 2,7 & 3,0 \\
\hline Mn oxalato $\left(\mathrm{g} \mathrm{kg}^{-1}\right)^{(6)}$ & 0,7 & 0,7 & 0,7 & 0,6 \\
\hline $\operatorname{Argila}\left(\mathrm{kg} \mathrm{kg}^{-1}\right)$ & 0,537 & 0,651 & 0,587 & 0,596 \\
\hline Umidade a $0,3 \mathrm{MPa}\left(\mathrm{kg} \mathrm{kg}^{-1}\right)^{(7)}$ & 0,546 & 0,269 & 0,269 & 0,368 \\
\hline Densidade do solo $\left(\mathrm{Mg} \mathrm{m}^{-3}\right)^{(8)}$ & 1,01 & 1,09 & 1,27 & 1,10 \\
\hline
\end{tabular}

${ }^{(1)} \mathrm{MO}$, matéria orgânica; (2) $\mathrm{SB}$, soma de bases; ${ }^{(3)} \mathrm{CTC}_{\mathrm{pH}}$ e $\mathrm{CTA}_{\mathrm{pH}}$, capacidade de troca de cátions e de ânions a $\mathrm{pH}$ do solo, respectivamente; ${ }^{(4)} \mathrm{CL}$, carga líquida (diferença entre CTA e CTC); ${ }^{(5)} \mathrm{PESN}$, ponto de efeito salino nulo; ${ }^{(6)} \mathrm{Fe}, \mathrm{Al}$ ou $\mathrm{Mn}$ oxalato, extraídos com oxalato de amônio; (7)umidade a $0,3 \mathrm{MPa}$, determinada em câmara de pressão; ${ }^{(8)}$ densidade, método do anel volumétrico, determinada em campo. 
foi registrada a concentração de nitrato no efluente em relação àquela aplicada inicialmente no pulso $\left(\mathrm{C} / \mathrm{C}_{0}\right)$. $\mathrm{O}$ total de nitrato recuperado (lixiviado) foi calculado como a área sob o gráfico das concentrações $\mathrm{C}$ em função do volume total (V) de soluções aplicadas. Essa área foi calculada pela regra trapezoidal, utilizando-se o programa computacional QtiPlot versão 0.9.0 (para ambiente Linux).

A equação convectiva-dispersiva (CDE) desenvolvida para quantificar os processos de transporte de solutos em experimentos conduzidos no laboratório em colunas de solo foi:

$\mathrm{FR} \frac{\partial \mathrm{C}}{\partial \mathrm{t}}=\mathrm{D} \frac{\partial^{2} \mathrm{C}}{\partial \mathrm{x}^{2}}-\frac{\partial \mathrm{C}}{\partial \mathrm{x}}$

em que C representa a concentração do soluto no líquido em movimento $\left(\mathrm{mol} \mathrm{m}^{-3}\right)$, $\mathrm{t}$ é o tempo (h), $\mathrm{x}$ é o deslocamento no sentido do fluxo (m). Essa equação considera ainda dois importantes parâmetros relacionados à movimentação, a saber: FR, fator de retardamento (adimensional); e $\mathrm{D}$, coeficiente de dispersão hidrodinâmica longitudinal, também denominado simplesmente de coeficiente de difusão $\left(\mathrm{m}^{2} \mathrm{~h}^{-1}\right)$. O FR pode ser entendido como a defasagem entre a velocidade de deslocamento do soluto e a do fluido carregador.

A solução da equação (1) implica assumir certas condições iniciais e de contorno. O limite inicial de entrada no sistema é $\mathrm{x}=0$. Considera-se também que o sistema é infinitamente longo na extensão da direção $+\mathrm{x}$. Existe uma concentração uniforme $\mathrm{C}_{\mathrm{i}}$ ao longo do sistema (solo da coluna) e, em um tempo inicial $\mathrm{t}=0$, um pulso contendo uma concentração $\mathrm{C}_{0}$ do soluto é introduzido na posição inicial $\mathrm{x}=0$ durante um certo tempo de aplicação $t_{p}$ (tempo de duração do pulso). Matematicamente, essas condições são expressas de acordo com as equações:

$\mathrm{C}(\mathrm{x}, \mathrm{t}=0)=\mathrm{C}_{\mathrm{i}}$

$\mathrm{C}(\mathrm{x}=0, \mathrm{t})=0 ; 0<\mathrm{t} \leq \mathrm{t}_{\mathrm{p}} ; \mathrm{C}(\mathrm{x} \rightarrow \infty, \mathrm{t})=\mathrm{C}_{\mathrm{i}}$

A solução da equação (1), sujeita às condições (2) e (3), pode ser derivada usando o princípio da superposição aplicado à solução para uma entrada contínua com as equações:

$$
\begin{aligned}
& C(x, t)=C_{i}+\left(C_{0}-C_{i}\right) \cdot A(x, t) t \leq t_{p} \\
& C(x, t)=C i\left(C_{0} \cdot C_{i}\right) \cdot\left[A(x, t)-A\left(x, t-t_{p}\right)\right] t>t_{p} \\
& A(x, t)=\frac{1}{2} \cdot\left[\operatorname{erfc}\left(\frac{x-\frac{v}{F R} \cdot t}{\sqrt{\frac{D}{F R}} \cdot t}\right)+\exp \left(\frac{v \cdot x}{D}\right) \cdot \operatorname{erfc}\left(\frac{x+\frac{v}{F R} \cdot t}{\sqrt{\frac{D}{F R}} \cdot t}\right)\right]
\end{aligned}
$$

em que: erfc() é a função do erro complementar, obtida subtraindo-se da unidade a função do erro: (1 - erf). A equação (1), com pequenas variações, tem sido utilizada por diferentes autores para descrever a movimentação de nitrato (e de outros íons) em experimentos de lixiviação em colunas de solo (Maraqa et al., 1998; Qafoku et al., 2000; Hutchison et al., 2003).

No presente trabalho, ajustou-se a solução da equação (1) aos dados experimentais das curvas de transposição. Para simular a movimentação desse elemento pelo solo, foi utilizado o aplicativo computacional STANMOD, versão 2.07 (Simunek et al., 2008). O modelo pede diversos parâmetros de entrada, determinados experimentalmente: comprimento da coluna (Z); velocidade (fluxo) do líquido nos poros (v), obtida dividindo-se o fluxo de Darcy pela porosidade do solo; concentração inicial do pulso $\left(\mathrm{C}_{0}\right)$; duração da aplicação do pulso $\left(\mathrm{t}_{\mathrm{p}}\right)$; intervalo de coleta de cada amostra no efluente $(\Delta t)$; e número de amostras que farão parte do ajuste $(\mathrm{N})$. Outros dois parâmetros são calculados automaticamente pelo modelo: fator de retardamento (FR) e coeficiente de dispersão-difusão (D). A adequação do modelo em descrever a movimentação foi avaliada calculando-se a significância do $\mathrm{F}$ da análise de correlação ( $\mathrm{p}$-value). $\mathrm{O}$ coeficiente de determinação $\left(\mathrm{R}^{2}\right)$ explica quanto da variação em uma das variáveis é devido à variação da outra variável (Regazzi, 2003). Assim, utilizou-se o R ${ }^{2}$ como uma estimativa da participação do modelo na predição dos valores de concentração relativa $\left(\mathrm{C} / \mathrm{C}_{0}\right)$ das curvas de eluição obtidas experimentalmente.

\section{Resultados e Discussão}

As densidades dos solos nas colunas utilizadas nos experimentos foram determinadas em ensaios prévios como sendo as resultantes da colocação do solo com leve compactação (Tabela 2). As densidades assim 
obtidas diferiram das originalmente encontradas em condições de campo (Tabela 1), em decorrência de alterações na estrutura originalmente encontrada no campo, ocasionadas pelas operações de secagem e peneiramento das amostras após a coleta. A porosidade variou de 0,49 a $0,56 \mathrm{~cm}^{3} \mathrm{~cm}^{-3}$ de solo, tendo sido calculada a partir das densidades do solo e de partículas. Uma vez que as colunas foram mantidas em condições de fluxo saturado, considerou-se que a umidade volumétrica no interior da coluna era igual à porosidade.

Em todas as colunas, o volume do pulso de nitrato aplicado foi igual a $100 \mathrm{~mL}$. Embora tenha sido utilizada uma bomba peristáltica para a aplicação do pulso e da solução deslocadora, não foi obtida uma densidade de fluxo igual em todas as colunas $(3,31$ a $\left.4,22 \mathrm{~cm}^{3} \mathrm{~cm}^{-2} \mathrm{~h}^{-1}\right)$. Pequenas variações na densidade de fluxo também foram encontradas por outros pesquisadores que trabalharam com lixiviação de nitrato em colunas de solo. Bellini et al. (1996) encontraram variação no fluxo semelhante à obtida aqui, entre 5,58 e $8,16 \mathrm{~cm}^{3} \mathrm{~cm}^{-2} \mathrm{~h}^{-1}$. Alcântara \& Camargo (2001), ao estudar a movimentação de cromo em colunas de solo sem o uso de bomba peristáltica para adição dos líquidos afluentes, trabalharam com variações no fluxo bastante superiores às encontradas aqui, sem que isso tenha afetado sobremaneira o formato das curvas de transposição.

As quantidades totais de nitrato recuperadas no efluente variaram de 0,405 a $1,432 \mathrm{mmol} \mathrm{L}^{-1}$ de $\mathrm{NO}_{3}{ }^{-}$ nos diferentes solos e horizontes utilizados (Figura 1). É interessante notar que a quantidade recuperada no LV-A (1,432 $\left.\mathrm{mmol} \mathrm{L}^{-1}\right)$ foi maior que o total aplicado durante o pulso $\left(1 \mathrm{mmol} \mathrm{L}^{-1}\right)$. Essa elevada quantidade de nitrato recuperada não é decorrente da lixiviação do nitrato previamente existente no solo. Antes de receber o pulso de nitrato, as colunas foram intensamente lixiviadas com solução deslocadora, livre desse íon.
Assim, uma hipótese para explicar o ocorrido relaciona-se com a metodologia de cálculo da área sob a curva do gráfico C vs. V. Esse cálculo é feito considerando-se separadamente as concentrações parciais de nitrato em cada volume parcial coletado no efluente. Portanto, o resultado final do cálculo seria afetado cumulativamente pelos erros parciais de quantificação. Ao menos teoricamente, esse erro poderia ser minimizado se, ao término da lixiviação e das respectivas análises, fosse determinada uma "concentração média" de nitrato no efluente, resultado da mistura total das quantidades de líquido coletadas em todos os tubos de ensaio, o que não foi possível, mas serve de alerta.

A quantificação dos teores totais recuperados tem sido empregada com sucesso para estudar a movimentação de diferentes solutos, como nos trabalhos com lixiviação de cromo de Alcântara \& Camargo (2001) e de Corrêa et al. (2005). No caso do nitrato, o total recuperado no efluente poderia ser uma boa avaliação da movimentação no solo em condições de lixiviação não muito intensas, como em experimentos realizados no campo, nos quais a remoção do nitrato é obtida pela percolação da água da chuva (em condições naturais ou simuladas). Entretanto, é necessário considerar que, conforme comentam Black \& Waring (1976) e Cantarella (2007), o ânion nitrato tem baixa interação química com o solo, e sua adsorção é do tipo eletrostática, não específica, com baixa afinidade energia de ligação - pelos sítios de adsorção.

Alcântara \& Camargo (2005) ajustaram os valores de $\mathrm{n}$ da equação de adsorção de Freundlich para os solos aqui utilizados. Os elevados valores obtidos de $n$, entre 3,066 a 4,383, indicam que existe baixa afinidade entre o nitrato e os sítios de adsorção dos solos. Isso indica que o nitrato é fracamente adsorvido no solo e é facilmente lixiviado. Assim, sob regime de lixiviação intensa, como o simulado neste estudo, pode-se esperar que o nitrato seja totalmente ou quase totalmente

Tabela 2. Propriedades dos solos Nitossolo Vermelho eutrófico (NV) e Latossolo Vermelho acriférrico (LV) nos horizontes A e B após a inserção nas colunas de lixiviação.

\begin{tabular}{|c|c|c|c|c|c|}
\hline Solo & Massa do solo & Densidade do solo & Porosidade & Fluxo $(\mathrm{Q})^{(1)}$ & $\mathrm{p}=\mathrm{V} / \mathrm{V}_{0}^{(2)}$ \\
\hline & $(\mathrm{kg})$ & $\left(\mathrm{Mg} \mathrm{m}^{-3}\right)$ & $\left(\mathrm{m}^{3} \mathrm{~m}^{-3}\right)$ & $\left(\mathrm{cm}^{3} \mathrm{~cm}^{-2} \mathrm{~h}^{-1}\right)$ & (adimensional) \\
\hline NV-A & 0,902 & 1,22 & 0,494 & 3,60 & 2,32 \\
\hline NV-B & 0,850 & 1,15 & 0,521 & 3,66 & 2,50 \\
\hline LV-A & 0,909 & 1,23 & 0,546 & 4,22 & 3,58 \\
\hline LV-B & 0,909 & 1,23 & 0,523 & 3,31 & 2,69 \\
\hline
\end{tabular}

${ }^{(1)}$ Fluxo em condições saturadas: a umidade volumétrica $(\theta)$ é igual à porosidade total do solo. ${ }^{(2)}$ Volumes de poros aplicados (p), obtido pela relação $\mathrm{p}=\mathrm{V} / \mathrm{V}_{0}$, onde $\mathrm{V}$ é o volume de pulso + solução deslocadora e $\mathrm{V}_{0}$ é o volume ocupado pelos poros na coluna. 
removido do solo. Consequentemente, a determinação das quantidades totais recuperadas não seria uma maneira adequada de avaliar o efeito dos diferentes tratamentos na movimentação desse ânion, ao menos nas condições experimentais aqui empregadas. Isso parece indicar a necessidade de se considerarem outros parâmetros e critérios para avaliar a lixiviação de nitrato em experimentos de colunas de solo. Assim, a aplicação de modelos matemáticos para predizer a movimentação vem ao encontro da busca desses parâmetros e critérios para estudo da movimentação.

Os parâmetros de entrada necessários para utilizar o modelo encontram-se na Tabela 3. A análise de variância da regressão entre os dados das curvas de transposição experimentais e ajustadas pelo modelo mostrou que os $\mathrm{F}$ foram significativos a $5 \%$ para as colunas NV-A, NV-B e LV-B, o que indica que a aplicação do modelo conseguiu descrever satisfatoriamente a movimentação do nitrato nesses solos (Tabela 4). Isso não ocorreu
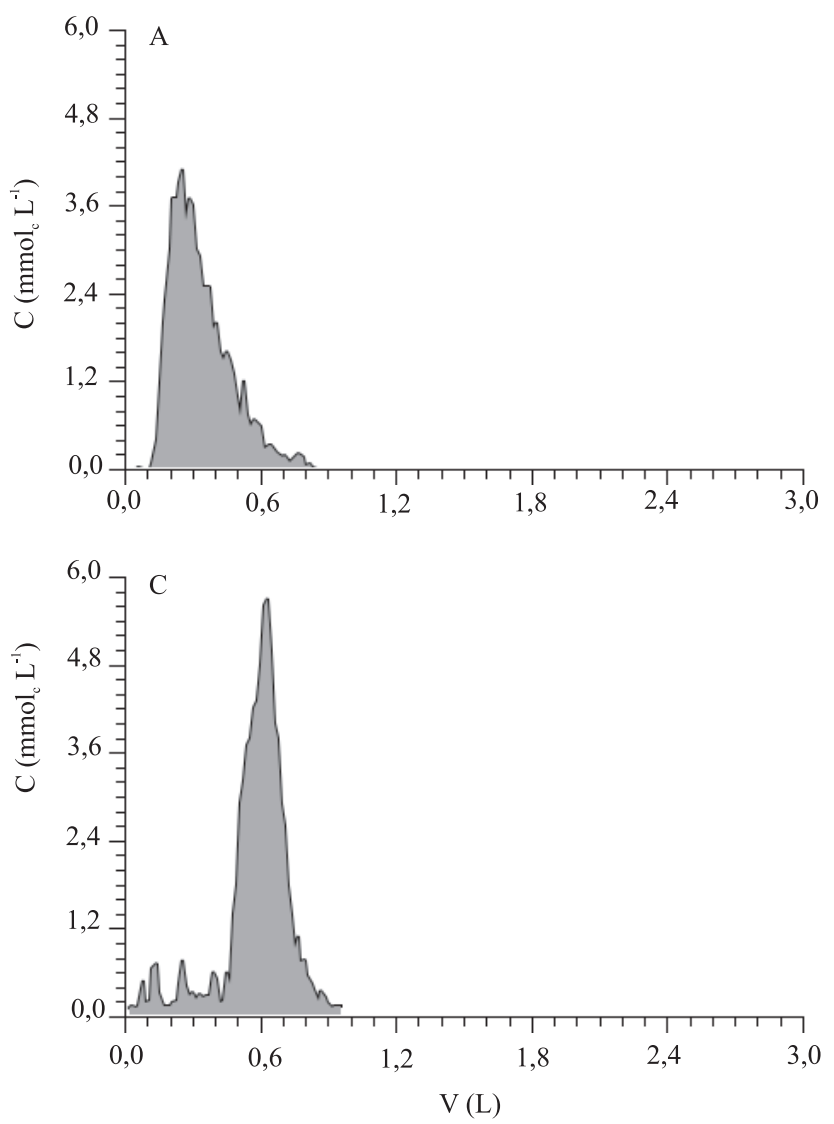

no LV-A. Nessa coluna, a análise de regressão da correlação apontou $\mathrm{F}$ não significativo a 5\%. O LV-A aqui utilizado apresenta maior quantidade de matéria orgânica em comparação aos outros solos (Tabela 1). Em vista disso, uma possível explicação para a falta de ajuste entre o modelo e os dados experimentais no LV-A poderia ser a de que o modelo não foi desenvolvido considerando a influência da matéria orgânica na movimentação de nitrato, embora isso esteja de certa forma embutido na grande dependência dos locais de carga às condições de $\mathrm{pH}$, força iônica e natureza do íon. Além disso, é possível que esteja ocorrendo exclusão iônica. Essa segunda hipótese não necessariamente exclui a anterior. $\mathrm{O}$ início da recuperação de nitrato em valores de $\mathrm{V} / \mathrm{V}_{0}$ inferiores a 1,00 verificado nessa coluna (Figura 2) corrobora a hipótese de exclusão iônica.

A coincidência entre as curvas experimentais e as ajustadas pelo modelo pode ser visualizada na
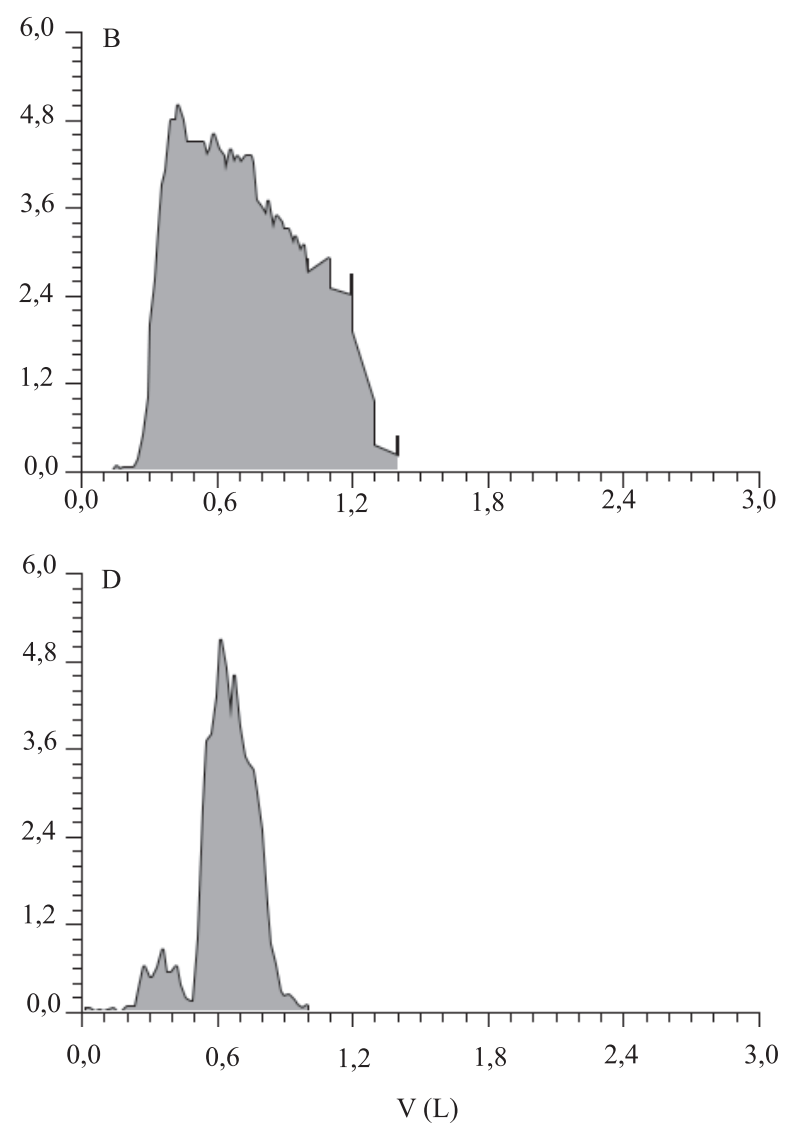

Figura 1. Quantidades totais de nitrato recuperado (C) em função do volume de pulso+solução deslocadora aplicado (V) nas colunas conduzidas para os solos Nitossolo Vermelho eutrófico (NV) e Latossolo Vermelho acriférrico (LV) nos horizontes A e B. A, NV-A: 0,405 mmol ; B, LV-A: 1,432 mmol $_{c}$; C, NV-B: 0,455 mmol ; e D, LV-B: 0,474 mmol . 
Figura 2. Os coeficientes de determinação elevados de 0,871 a 0,955 para NV-A, NV-B e LV-B indicam que grande parte da variação nos valores das concentrações relativas experimentais $\mathrm{C} / \mathrm{C}_{0}$ puderam ser previstas pelo ajuste do modelo. No caso do LV-A, o modelo respondeu por apenas $23,4 \%$ desses valores.

O modelo fornece dois importantes parâmetros de transporte, o FR e o D (Tabela 4). Quando o modelo se ajusta aos dados experimentais, entende-se que ele

Tabela 3. Parâmetros de entrada para o modelo obtidos para os solos Nitossolo Vermelho eutrófico (NV) e Latossolo Vermelho acriférrico (LV) nos horizontes A e B dos solos ${ }^{(1)}$.

\begin{tabular}{lccccc}
\hline Solo & $\begin{array}{c}\mathrm{C}_{0} \\
\left(\mathrm{mmol}_{\mathrm{c}} \mathrm{L}^{-1}\right)\end{array}$ & $\begin{array}{c}v \\
\left(\mathrm{~cm} \mathrm{~h}^{-1}\right)\end{array}$ & $\begin{array}{c}\mathrm{t}_{\mathrm{p}} \\
(\mathrm{h})\end{array}$ & $\begin{array}{c}\mathrm{x} \\
(\mathrm{cm})\end{array}$ & $\begin{array}{c}\mathrm{N}^{\mathrm{o}} \mathrm{de} \\
\text { Pontos }\end{array}$ \\
\hline NV-A & 10 & 7,28 & 1,17 & 30 & 56 \\
NV-B & 10 & 6,91 & 1,11 & 30 & 64 \\
LV-A & 10 & 6,59 & 1,18 & 30 & 83 \\
LV-B & 10 & 6,88 & 1,11 & 30 & 52 \\
\hline
\end{tabular}

${ }^{(1)} \mathrm{C}_{0}$, concentração inicial do pulso; $\mathrm{v}$, velocidade (fluxo) do líquido nos poros, obtida dividindo-se o fluxo de Darcy pela porosidade do solo; $t_{p}$, duração da aplicação do pulso; x, comprimento da coluna. consegue descrever a movimentação do soluto pelo solo (Zheng \& Bennett, 2002). Nesse caso, é razoável supor que os parâmetros de ajuste D e FR fornecidos pelo modelo quantifiquem adequadamente alguns fenômenos importantes dessa movimentação. Nas colunas com bom ajuste entre dados experimentais e preditos, os FR variaram de $1,055 \pm 0,009$ a $2,747 \pm 0,001$. O fato de

Tabela 4. Significância da análise de regressão (p) entre os dados experimentais e ajustados, fatores de retardamento (FR) e coeficientes de dispersão-difusão (D) fornecidos pela aplicação do modelo aos solos Nitossolo Vermelho (NV) e Latossolo Vermelho (LV), horizontes A e B.

\begin{tabular}{cccc}
\hline Solos & Probabilidade $^{(1)}$ & $\mathrm{D}$ & FR \\
\hline & & $\left(\mathrm{cm}^{2} \mathrm{~h}^{-1}\right)$ & \\
NV-A & 0,272 & $6,168 \pm 0,382$ & $1,055 \pm 0,009$ \\
NV-B & 0,482 & $1,770 \pm 0,106$ & $1,454 \pm 0,006$ \\
LV-A & 0,072 & $11,64 \pm 4,79$ & $1,223 \pm 0,105$ \\
LV-B & 0,226 & $1,83 \pm 0,237$ & $2,747 \pm 0,001$ \\
\hline
\end{tabular}

(1)Probabilidade de obter-se resultados fora da região de possibilidades de conclusão pela análise de variância da regressão. Se $p<0,05$, a correlação entre os dados experimentais e simulados pelo modelo é significativa a $5 \%$.
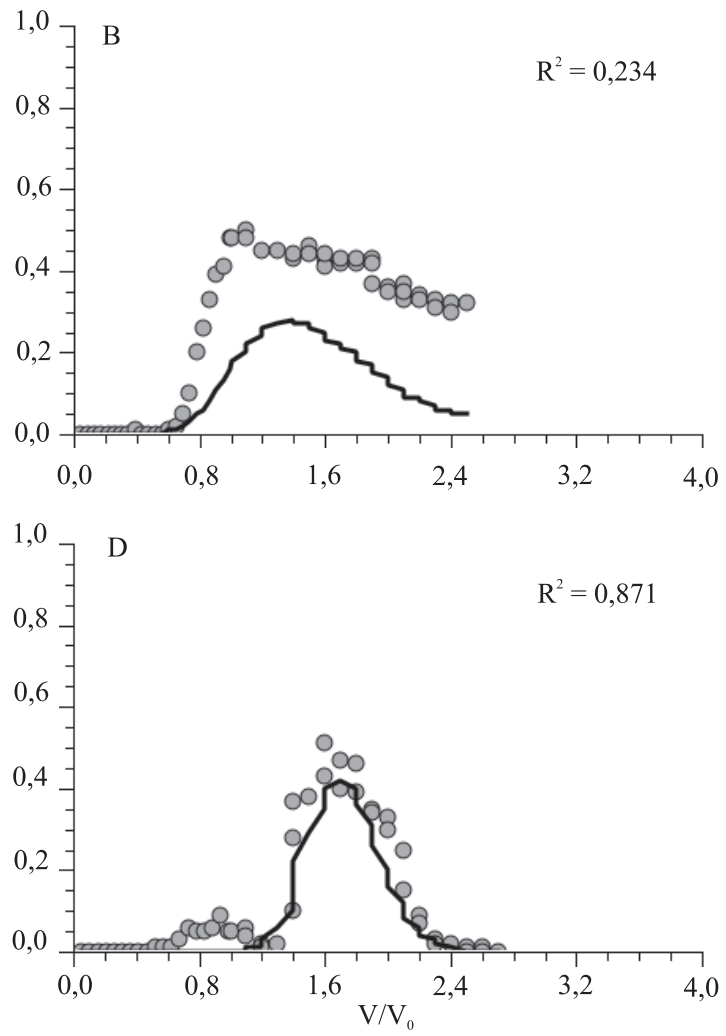

Figura 2. Curvas de transposição para os solos e horizontes estudados: A, Nitossolo, horizonte A; B, Latossolo, horizonte A; C, Nitossolo, horizonte B; e D, Latossolo, horizonte B. Pontos: dados obtidos experimentalmente; linha contínua: ajuste do modelo. C/Co: concentração relativa; V/Vo: volume de poros de pulso+solução deslocadora aplicado. $\mathrm{R}^{2}$ : coeficiente de determinação entre os dados experimentais e ajustados pelo modelo. 
todos os fatores de retardamento terem sido maiores que 1,0 indica que a movimentação do nitrato foi retardada em relação ao avanço da frente de molhamento do solo. Considerando que a adsorção eletrostática de ânions em solos de cargas variáveis é causada pela força de atração entre a carga superficial positiva do solo e os ânions, esperase que essa adsorção esteja fortemente correlacionada às quantidades de cargas positivas, com sua consequente densidade de cargas (Ji, 1997). Bellini et al. (1996) e McVay et al. (2004) mostraram que quanto maior a CTA, maior o retardamento e menor a movimentação do ânion. Como a adsorção do nitrato é predominantemente eletrostática, é de se esperar que, nas cargas positivas do solo, quanto maior a quantidade de cargas positivas de um solo, maior o FR. No presente trabalho, se for excluído dessa discussão o FR do LV-A devido à falta de ajuste do modelo aos dados experimentais, observa-se claramente que o maior valor de FR foi obtido para o LV-B, justamente o solo que possui a maior quantidade de cargas positivas (CTA igual a 7,8 mmol $_{\mathrm{c}} \mathrm{dm}^{-3}$ ) em comparação com o NV-A e NV-B (CTA iguais a 5,1 e 4,8 $\mathrm{mmol}_{\mathrm{C}} \mathrm{dm}^{-3}$, respectivamente).

Finalmente, cabe lembrar que, em princípio, a extrapolação direta dos resultados de colunas de lixiviação para condições de campo deve ser evitada, por se tratar de situações distintas em relação aos pressupostos teóricos e experimentais envolvidos, ou, pelo menos, extrapolações devem ser feitas com muita prudência.

\section{Conclusões}

1. As quantidades lixiviadas de nitrato recuperadas nas colunas dos solos Nitossolo Vermelho eutrófico e Latossolo Vermelho acriférrico dos horizontes A e B são de 0,405 a $1,432 \mathrm{mmol} \mathrm{L}^{-1}$.

2. O modelo matemático utilizado é adequado para prever a lixiviação de nitrato em colunas do horizonte B dos solos Nitossolo Vermelho eutrófico e Latossolo Vermelho acriférrico.

3. Nas colunas dos solos com bom ajuste entre dados experimentais e preditos, o fator de retardamento é maior do que 1 , o que indica que a movimentação do nitrato é retardada em relação ao avanço da frente de molhamento do solo.

\section{Referências}

ALCÂNTARA, M.A.K. de; CAMARGO, O.A. de. Adsorção de nitrato em solos com cargas variáveis. Pesquisa Agropecuária Brasileira, v.40, p.369-376, 2005.
AlCÂNTARA, M.A.K. de; CAMARGO, O.A. de. Chromium movement in columns of two highly weathered soils. Communications in Soil Science and Plant Analysis, v.35, p.599-613, 2004.

ALCÂNTARA, M.A.K. de; CAMARGO, O.A. de. Transporte de crômio trivalente influenciado pelo $\mathrm{pH}$, horizonte do solo e fontes do crômio. Revista Brasileira de Engenharia Agrícola e Ambiental, v.5, p.497-501, 2001.

BAKER, A.S. Colorimetric determination of nitrate in soil and plant extracts with brucine. Journal of Agricultural and Food Chemistry, v.15, p.802-806, 1967.

BARIZON, R.R.M.; LAVORENTI, A.; REGITANO, J.B.; PRATA, F.; TORNISIELO, V.L. Simulação do transporte e da sorção de imazaquim em colunas de solo. Revista Brasileira de Ciência do Solo, v.30, p.615-623, 2006.

BELLINI, G.; SUMNER, M.E.; RADCLIFFE, D.E.; QAFOKU, N.P. Anion transport through columns of highly weathered acid soils: adsorption and retardion. Soil Science Society of American Journal, v.60, p.132-137, 1996.

BLACK, A.S.; WARING, S.A. Nitrate leaching and adsorption in a Krasnozem from Redland Bay, Qld. I. Leaching of banded ammonium nitrate in a horticultural rotation. Australian Journal of Soil Research, v.14, p.171-180, 1976.

BORGES JÚNIOR, J.C.F.; FERREIRA, P.A. Equações e programa computacional para cálculo do transporte de solutos do solo. Revista Brasileira de Engenharia Agrícola e Ambiental, v.10, p.604-611, 2006.

CAMARGO, O.A. de; MONIZ, A.C.; JORGE, J.A.; VALADARES, J.M.A.S. Métodos de análise química, mineralógica e física de solos do Instituto Agronômico de Campinas. Campinas: Instituto Agronômico, 1986. 94p. (IAC. Boletim técnico, 106).

CANTARELLA, H. Nitrogênio. In: NOVAIS, R.F.; ALVAREZ V.,V.H.; BARROS, N.F. de; FONTES, R.L.F.; CANTARUTTI, R.B.; NEVES, J.C.L. (Ed.). Fertilidade do solo. Viçosa: Sociedade Brasileira de Ciência do Solo, 2007. p.375-470.

CORRÊA, R.S.; WHITE, R.E.; WEATHERLEY, A.J. Modeling the risk of nitrate leaching from two soils amended with five different biosolids. Revista Brasileira de Ciência do Solo, v.29, p.619-626, 2005.

DOLTRA, J.; MUÑHOZ, P. Simulation of nitrogen leaching from a fertigated crop rotation in a Mediterranean climate using the EU-Rotate_N and Hydrus-2D models. Agricultural Water Management, v.97, p.277-285, 2010.

DONN, M.J.; MENZIES, N.W. The effect of ionic strength variation and anion competition on the development of nitrate accumulations in variable charge subsoils. Australian Journal of Soil Research, v.43, p.43-50, 2005.

FONSECA, B.; TEIXEIRA, A.; FIGUEIREDO, H.; TAVARES, T. Modeling of the $\mathrm{Cr}(\mathrm{VI})$ transport in typical soils of the North of Portugal. Journal of Hazardous Materials, v.167, p.756-762, 2009 .

HUTCHISON, J.M.; SEAMAN, J.C.; ABURIME, S.A.; RADCLIFFE, D.E. Chromate transport and retention in 
variably saturated soil columns. Vadose Zone Journal, v.2, p.702-714, 2003.

JI, G.L. Electrostatic adsorption of anions. In: YU, T.R. (Ed.). Chemistry of variable charge soils. Oxford: Oxford University, 1997. p.112-139.

MARAQA, M.A.; ZHAO, X.; WALLACE, R.B.; VOICE, T.C. Retardation coefficients of nonionic organic compounds determined by batch and column techniques. Soil Science Society of America Journal, v.62, p.142-152, 1998.

MCVAY, K.A.; RADCLIFFE, D.E.; WEST, I.T.; CABRERA, M.L. Anion exchange in saprolite. Vadose Zone Journal, v.3, p.668-675, 2004.

MELO, R.F. de; FERREIRA, P.A.; MATOS, A.T. de.; RUIZ, H.A.; OLIVEIRA, L.B. de. Deslocamento miscível de cátions básicos provenientes da água residuária de mandioca em colunas de solo. Revista Brasileira de Engenharia Agrícola e Ambiental, v.10, p.456-465, 2006.

OLIVEIRA, F.C.; MATTIAZZO, M.E.; MARCIANO, C.R.; MORAES, S.O. Lixiviação de nitrato em um Latossolo Amarelo distrófico tratado com lodo de esgoto e cultivado com cana-de-açúcar. Scientia Agricola, v.58, p.171-180, 2001.

PRATA, F.; LAVORENTI, A.; VANDERBORGHT, J.; BURAUEL, P.; VEREECKEN, H. Miscible displacement, sorption and desorption of atrazine in a Brazilian Oxisol. Vadose Zone Journal, v.2, p.728-738, 2003.
QAFOKU, N.P.; SUMNER, M.E.; RADCLIFFE, D.E. Anion transport in columns of variable charge subsoils: nitrate and chloride. Journal of Environmental Quality, v.29, p.484-493, 2000.

REGAZZI, A.J. Teste para verificar a igualdade de parâmetros e a identidade de modelos de regressão não-linear. Revista Ceres, v.50, p.9-23, 2003.

REICHARDT, K.; TIMM, L.C. Solo, planta e atmosfera: conceitos, processos e aplicações. Barueri: Manole, 2004. 478p.

ROSE, D.A.; ABBAS, F.; ADEY, M.A. The effect of surface solute interactions on the transport of solutes through porous materials. European Journal of Soil Science, v.60, p.398-411, 2009.

SANTOS, H.G. dos; JACOMINE, P.K.T.; ANJOS, L.H.C. dos; OLIVEIRA, V.A. de; OLIVEIRA, J.B. de; COELHO, M.R.; LUMBRERAS, J.F.; CUNHA, T.J.F. (Ed.). Sistema brasileiro de classificação de solos. Rio de Janeiro: Embrapa Solos, 2006. 306p.

SIMUNEK, J.; GENUCHTEN, M.T. van; SEJNA, M. Development and applications of the HYDRUS and STANMOD software packages and related codes. Vadose Zone Journal, v.7, p.587-600, 2008.

TRIVEDI, J.; BABADAGLI, T. Scaling miscible displacement in fractured porous media using dimensionless groups. Journal of Petroleum Science and Engineering, v.61, p.58-66, 2008.

ZHENG, C.; BENNETT, G.D. Applied contaminant transport modeling. $2^{\text {nd }}$ ed. New York: John Wiley \& Sons. 2002. 621p.

Recebido em 2 de novembro de 2008 e aprovado em 11 de dezembro de 2009 